\title{
Seven Autophagy-Related Genes are Associated with the Tumor Immune Microenvironment in Predicting Survival Risk of Non-Small Cell Lung Cancer
}

\section{Huihui Jiang}

First Affiliated Hospital of Anhui Medical University

\section{Aiqun $\mathrm{Xu}$}

Hefei Second People's Hospital

Min Li

First Affiliated Hospital of Anhui Medical University

Rui Han

First Affiliated Hospital of Anhui Medical University

\section{Enze Wang}

First Affiliated Hospital of Anhui Medical University

\section{Ke Zhu}

First Affiliated Hospital of Anhui Medical University

\section{Sijing Zhou}

hefei prevention and treatment center for occupational diseases

ran wang ( $\boldsymbol{\nabla}$ wangran@ahmu.edu.cn )

First Affiliated Hospital of Anhui Medical University https://orcid.org/0000-0003-2069-2887

\section{Primary research}

Keywords: non-small cell lung cancer, autophagy, LncRNA, immune microenvironment

Posted Date: May 21st, 2021

DOI: https://doi.org/10.21203/rs.3.rs-508914/v1

License: (c) (1) This work is licensed under a Creative Commons Attribution 4.0 International License. Read Full License 


\section{Abstract}

Background: Non-small cell lung cancer (NSCLC) ranks first among global cancer-related deaths. Despite the emergence of various immunological and targeted therapies, immune tolerance remains a barrier to treatment.

Methods: It has been found that this obstacle can be overcome by targeting autophagy-related genes (ATGs). ATGs were screened by coexpression analysis and the genes related to the prognosis of lung cancer were screened using Kaplan-Meier (K-M) survival analysis, univariate Cox regression, and multivariate Cox regression. The prognostic risk model of ATGs was constructed and verified using K-M survival analysis and receiver operating characteristic (ROC) curve analysis.

Results: The prognostic risk model of ATGs was constructed. Gene set enrichment analysis (GSEA) showed that the function and pathway of ATG enrichment were closely related to immune cell function. CIBERSORT, LM22 matrix, and Pearson correlation analysis showed that risk signals were significantly correlated with immune cell infiltration and immune checkpoint genes.

Conclusions: We identified and independently verified the ATG (AL691432.2, MMP2-AS1, AC124067.2, CRNDE, ABALON, AL161431.1, NKILA) in NSCLC patients and found that immune regulation in the tumor microenvironment is closely related to this gene.

\section{Introduction}

Owing to its rapid morbidity and mortality, lung cancer poses a great challenge to human health and life on a global scale [1]. The 2017 Global Burden of Disease Study reveals that there are 2.2 million new cases of lung cancer and 1.9 million deaths annually [2]. Non-small cell lung cancer (NSCLC) accounts for approximately $85 \%$ of lung cancers $[3,4]$, making it the leading cause of cancer-related deaths worldwide. The current treatment methods for lung cancer include surgery, chemoradiotherapy, and targeting therapy; however, NSCLC has strong aggressiveness and heterogeneity, drug resistance, recurrence, and even malignant progression. Therefore, new treatment options are urgently needed. Immunotherapy is currently carried out in a variety of cancers. As immunotherapy aims to arouse the tumor recognition function of the patient, this is also a recent research direction for lung cancer. Given that immune tolerance currently presents an obstacle in this research, we hope to find new biomarkers to guide treatment.

Autophagy is an "eat yourself" phenomenon that can use the components of a cell to recompose other required components. In normal cells, autophagy helps maintain homeostasis. In the context of starvation or malignancy, significantly upregulated autophagy functions [5] can maintain the metabolic requirements of cells [6-8]. Autophagy can reduce cell death, damage, and chronic inflammation caused by the accumulation of harmful substances in cells during stress [9]. An increasing number of studies have shown that autophagy is also involved in immune regulation. In previous studies on renal carcinoma, it was found that the immune efficacy of tumors could be enhanced by targeting autophagy. 
Similar findings were also found in studies on oral squamous cell carcinoma. However, autophagy has two functions in cancer [10]. Tumor cells can be more tolerant to hypoxia [11], starvation, and treatment through autophagy; however, autophagy can inhibit tumor growth and proliferation by limiting chronic cell death and inducing chronic tumor inflammation. Thus, autophagy could be used as a new method to treat or prevent cancer.

Considering the two-sided nature of autophagy, it is very important to evaluate and search for prognostic biomarkers that can precisely regulate this process. The purpose of this study was to identify long noncoding RNAs (IncRNAs) related to the prognosis of NSCLC and to predict the efficacy of immunotherapy through immune microenvironment analysis.

\section{Material And Methods}

\subsection{Data download and collation}

We downloaded the genetic data of 1,146 NSCLC patients (including 1,038 cancer samples and 108 paracancerous samples) and 1,028 clinical data from The Cancer Genome Atlas (TCGA) database (https://portal.gdc.cancer.gov/). To improve the accuracy of the study, we excluded patients with a survival time of less than 30 days [12].

\subsection{Screening of autophagy-related IncRNAs}

Autophagy-related genes (ATGs) were downloaded from the Human Autophagy Database (HADb, (http://www.autophagy.lu/index.html). We used the "limma R" (http://www.bioconductor.org/) software package and the function of Cor in R (https://cran.r-project.org/) and set the coefficient of cor-filter to > 0.4 with $\mathrm{P}<0.001$. We analyzed the correlation between autophagy genes and the IncRNA expression level in the sample and determined the autophagy-related IncRNA.

\subsection{Prognosis model development}

The "Survival R" package was used to calculate survival prognosis. First, the Kaplan-Meier (K-M) test and univariate Cox regression analysis were used to screen IncRNAs with statistical significance. Then, multivariate Cox regression was used to screen IncRNAs with independent prognostic significance. Hazard ratios (HRs) were used to distinguish between high-risk IncRNAs (HR $>1)$ and protective IncRNAs $(H R<1)$. The risk score of each NSCLC patient was calculated based on the expression of the model IncRNA and its coefficient. The risk score was calculated as follows: risk score $=\beta$ gene $1 \times$ expressed gene $1+\beta$ gene $2 \times$ expressed gene $2+\beta$ gene $3 \times$ expressed gene $3+\ldots+\beta$ gene $7 \times$ expression gene 7 . Finally, patients with NSCLC were classified into high-risk and low-risk groups based on the median risk score.

\subsection{Verifying and evaluating the prognostic signature}

We used the K-M "Survival R" package and R software to draw the K-M survival curve. The K-M survival curve was used to calculate the difference in OS between the high- and low-risk groups. The receiver 
operating characteristic (ROC) curve was used to measure the predictive efficiency of various clinical indicators. A stratified survival analysis was then performed to determine the accuracy of the prognostic model. Finally, univariate and multivariate Cox regression analyses were performed to test whether the prognostic model was an independent risk factor. Statistical significance was set at $P<0.05$.

\subsection{Establishment and evaluation of nomograms}

Traditional clinical variables (such as age, sex, and AJCC stage) and risk scores derived from prognostic markers were used to construct a nomogram, and the OS of patients with NSCLC at 1, 3, and 5 years were analyzed. At the same time, a calibration curve was constructed to analyze the accuracy of the nomogram.

\subsection{Construction of the IncRNA-mRNA coexpression network.}

A IncRNA-mRNA coexpression network was constructed to analyze the correlation between autophagyrelated IncRNAs and target mRNAs. Cytoscape software (version 3.7.1, http://www.cytoscape.org/) was used to construct and visualize the IncRNA-mRNA coexpression network.

\subsection{Functional enrichment analysis}

Gene ontology (GO) enrichment analysis and Kyoto Encyclopedia of Genes and Genome (KEGG) pathway analysis were used to determine the mRNAs related to IncRNAs. Statistical significance was set at $\mathrm{P}<$ 0.05 .

\subsection{Gene Set Enrichment Analysis (GSEA)}

Gene set enrichment analysis (GSEA4.1.0) was performed on the whole genome expression profile of NSCLC patients to determine the biological functions of differentially expressed genes between high-risk and low-risk groups. We used 500 and 15 genes as the largest and smallest gene set sizes to filter gene sets, respectively. Permutation was performed 1,000 times.

\subsection{Analysis of immune cell components}

CIBERSORT is a robust and novel method that can construct the cellular composition of tissues (such as solid tumors) based on gene expression profiles. The LM22 matrix is a leukocyte gene marker matrix consisting of 547 genes that can distinguish 22 subtypes of human hematopoietic cells. CIBERSORT combined with the LM22 matrix was used to assess the composition of immune cells in the high-risk and low-risk groups according to the risk score. NSCLC samples were deleted $(P>0.05)$. At the same time, the correlation of immune cells was examined, and the correlation between each immune cell and risk score was calculated using the Pearson correlation method. The expression of classic immune checkpoint genes (PD-1, PD-L1, CTLA4, and ID01), which have traditionally been regarded as targets of immunotherapy, was studied.

\subsection{Statistical methods}


All statistical tests were conducted in $\mathrm{R} . \mathrm{P}<0.05$ was considered statistically significant.

\section{Results}

\subsection{Identification of autophagy-related IncRNAs with prognostic significance in tissue samples from NSCLC patients}

A total of 14,142 IncRNAs were identified from RNA sequencing (RNA-Seq) data of tissue samples from NSCLC patients downloaded from TCGA database, and 232 ATGs were extracted from the analysis of the HADb. Then, Pearson correlation analysis was performed between 14,142 IncRNAs and ATGs, and 1,495 autophagy-related IncRNAs were identified (Pearson $\mathrm{R}>0.4, \mathrm{P}<0.05$ ). Univariate and multivariate Cox regression analyses of 1,495 autophagy-related IncRNAs showed that seven types of IncRNAs were significantly related to the survival of NSCLC patients (AL691432.2, MMP2-AS1, AC124067.2, AL691432.2, MMP2-AS1, AC124067.2, CRNDE, ABALON, AL161431.1, and NKILA) ( $P<0.05$; Fig. 1, Supplementary Fig. 1). ABALON, AL161431.1, and NKILA are considered to be risk factors with HR values greater than 1, while the remaining four IncRNAs (AL691432.2, MMP2-AS1, AC124067.2, and CRNDE) were considered as protective factors with HR values less than 1 .

\subsection{Evaluation of prognostic markers including seven autophagy-related IncRNAs}

The risk score of each patient in TCGA dataset was calculated using the following formula to calculate the autophagy-related IncRNA signature: risk score $=(-0.096 \times$ AL691432.2 expression level $)+(-0.216 \times$ MMP2-AS1 expression level $)+(-0.045 \times$ AC124067 .2 expression level $)+(-0.020 \times$ CRNDE expression level $)+(0.351 \times$ ABALON expression level $)+(0.006 \times$ AL161431.1 expression level $)+(0.091 \times$ NKILA expression level). Using the median value of the risk score as the cutoff point, NSCLC patients were divided into high-risk $(n=466)$ and low-risk $(n=462)$ groups. K-M survival curve analysis showed that the overall survival (OS) and progression-free survival (PFS) of NSCLC patients with high-risk scores were significantly shorter than those of NSCLC patients with low-risk scores (Fig. 2A). The 3-year survival rates of high-risk and low-risk patients were $53.40 \%$ and $67.2 \%$, and the 5 -year survival rates were $35.34 \%$ and $50.1 \%$, respectively. Principal component analysis (PCA) based on seven autophagy-related IncRNAs showed that there were two distinct distribution patterns between the high-risk and low-risk groups (Fig. 2B). Time-dependent ROC curve analysis showed that the $1-, 3-$, and 5-year survival rates predicted by the three IncRNA risk score curves (AUC) were 0.660 (Fig. 2F), 0.641 (Fig. 2G), and 0.634 (Fig. 2H), respectively. The NSCLC patients were then ranked according to the risk score calculated using autophagy-related IncRNA prognostic indicators (Fig. 2C). The scatter plot shows that the survival rate of NSCLC patients was related to the risk score; patients with a higher risk score had a shorter survival time (Fig. 2D). The heat map shows that the expression levels of the seven prognostic marker-related IncRNAs were significantly different in NSCLC patients in different risk groups. High-risk patients expressed higher 
levels of risk factors (ABALON, AL161431.1, and NKILA), and low-risk patients expressed higher levels of protective factors (AL691432.2, MMP2-AS1, AC124067.2, and CRNDE) (Fig. 2E).

\subsection{Correlation analysis between autophagy-related IncRNA prognostic signals and other clinicopathological parameters}

We then analyzed the correlation between the risk score of the autophagy-associated IncRNA prognostic signature and the clinicopathological characteristics of NSCLC patients. We found that the risk scores were statistically similar among patients at all levels (Table 1). We further performed a stratified analysis to study the prognostic value of autophagy-related IncRNAs. Hierarchical analysis was performed according to the pathological classification, age ( $\leq 65$ years old, $>65$ years old), gender (male and female), AJCC stage (stage I, II and III, IV), T stage (T1/T2 and T3/T4), N stage (N0 and N1/N2/N3), and $\mathrm{M}$ stage (M0 and M1). As shown in Fig. 3, K-M survival curve analysis showed that the OS of patients with a high risk score in lung squamous cell carcinoma (LUSC), lung adenocarcinoma (LUAD), age > 65 years, male sex, and AJCC stage was shorter than that of patients with low risk scores, and the difference was statistically significant. We analyzed the PFS of patients and reached similar conclusions (Supplementary Fig. 2). This indicates that the prognostic characteristics can accurately judge the prognosis of patients compared with other clinicopathological characteristics. 
Table 1

Correlation analysis between prognostic markers of autophagy-related IncRNA and various clinicopathological features of NSCLC patients.

\begin{tabular}{|c|c|c|c|c|c|c|}
\hline Clinical & Group & $\mathrm{n}$ & Mean & SD & $t$ & P \\
\hline \multirow[t]{2}{*}{ Pathological type } & LUSC & 387 & 1.077 & 0.544 & \multirow[t]{2}{*}{-1.77682} & \multirow[t]{2}{*}{0.076} \\
\hline & LUAD & 325 & 1.24 & 1.579 & & \\
\hline \multirow[t]{2}{*}{ age } & $<=65$ & 312 & 1.159 & 1.577 & \multirow[t]{2}{*}{0.147977} & \multirow[t]{2}{*}{0.882} \\
\hline & $>65$ & 400 & 1.145 & 0.619 & & \\
\hline \multirow[t]{2}{*}{ gender } & FEMAL & 263 & 1.102 & 0.537 & \multirow[t]{2}{*}{-1.07784} & \multirow[t]{2}{*}{0.282} \\
\hline & MALE & 449 & 1.181 & 1.377 & & \\
\hline \multirow[t]{2}{*}{ stage } & Stage I-II & 557 & 1.138 & 1.259 & \multirow[t]{2}{*}{-0.91666} & \multirow[t]{2}{*}{0.36} \\
\hline & Stage III-IV & 155 & 1.201 & 0.54 & & \\
\hline \multirow[t]{2}{*}{$\mathrm{T}$} & T1-2 & 597 & 1.145 & 1.218 & \multirow[t]{2}{*}{-0.50403} & \multirow[t]{2}{*}{0.615} \\
\hline & T3-4 & 115 & 1.183 & 0.604 & & \\
\hline \multirow[t]{2}{*}{ M } & MO & 685 & 1.149 & 1.161 & \multirow[t]{2}{*}{-0.82439} & \multirow[t]{2}{*}{0.414} \\
\hline & M1 & 27 & 1.224 & 0.411 & & \\
\hline \multirow[t]{2}{*}{$N$} & NO & 447 & 1.091 & 0.584 & \multirow[t]{2}{*}{-1.506} & \multirow[t]{2}{*}{0.133} \\
\hline & N1-3 & 265 & 1.254 & 1.708 & & \\
\hline
\end{tabular}

\subsection{Autophagy-related IncRNA signals are independent prognostic factors}

Univariate and multivariate Cox regression analyses were performed to determine whether autophagyrelated IncRNA prognostic markers were independent prognostic factors for NSCLC patients. Univariate analysis showed that AJCC stage $(P<0.001)$, T stage $(P<0.001)$, $N$ stage $(P<0.001)$, $M$ stage $(P=0.02)$, and autophagy-related IncRNA prognostic risk score $(P<0.001)$ were significantly correlated with OS (Fig. 4A). Multivariate analysis showed that the autophagy-related IncRNA prognostic risk score $(\mathrm{P}<$ 0.001) was significantly correlated with OS (Fig. 4B). ROC curve analysis (Fig. 2F) showed that the AUC value of prognostic markers of autophagy-related IncRNAs was 0.660 -fold higher than that of age (AUC = $0.519)$, gender $(A \cup C=0.548), A J C C$ stage $(A \cup C=0.655)$, T stage $(A \cup C=0.634), N$ stage $(A U C=0.598)$, and $M$ stage (AUC $=0.502)$. These data indicate that autophagy-related IncRNA prognostic signals are independent prognostic factors for patients with NSCLC.

\subsection{Assessment of prognosis prediction nomogram, including autophagy-related IncRNA prognostic signature}




\section{risk score}

We used the risk score calculated by autophagy-related IncRNA prognostic signals and other clinicopathological factors (including age, gender, grade, AJCC stage, T stage, and N stage) to construct a nomogram to accurately estimate the 1-, 3-, and 5-year survival rates (Fig. 5A). The analysis of the calibration curve shows that the actual and predicted 1-, 3-, and 5-year survival times were similar (Figs. 5B-5D). These results indicate the reliability and accuracy of the nomogram, which contains the autophagy-related IncRNA prognostic signature risk score.

\subsection{Construction and functional enrichment analysis of the IncRNA-mRNA coexpression network}

The IncRNA-mRNA coexpression network was constructed using Cytoscape, which contained 28 pairs of IncRNA-mRNA (Pearson correlation coefficient $|\mathrm{R}|>0.4, \mathrm{P}<0.05$ ) (Fig. 6A). The Sankey diagram shows the relationship (risk/protection) between 28 types of mRNAs and seven types of IncRNAs (Fig. 6B). The first three $\mathrm{GO}$ terms of biological processes are autophagy, processes that utilize the autophagy mechanism, and macroautophagy (Fig. 6C). The first three GO terms for cellular components were autophagy, vacuolar membrane, and phosphor assembly site (Fig. 6D). The first three $\mathrm{GO}$ terms for molecular functions were protein serine/threonine kinase activity, microtubule binding, and tubulin binding (Fig. 6E). KEGG pathway analysis confirmed that autophagy was the most significantly enriched pathway (Fig. 6F).

\subsection{Gene set enrichment analysis (GSEA)}

To study the biological functions and pathways of ATG risk signals in NSCLC patients, we conducted GSEA. A total of 5,472 GO functions (the first 50 in Table S1) and 178 KEGG pathways (the first 50 in Table S2) were enriched, with many results related to cancer behavior, immune cell function, and response (adjusted P-value $<0.25$ ). The results of $G O$ and KEGG analyses showed that risk signals were closely related to the tumor immune microenvironment (Fig. 7A) and warranted further analysis.

\subsection{Recognition of immune cell landscape and its correlation with prognostic markers of ATGs}

Combining the CIBERSORT and LM22 matrix, as shown in the heat map of the immune cell distribution of the NSCLC sample (Fig. 7B), the bar graph (Supplementary Fig. 3), and the violin graph (Fig. 7C), showed that many immune cell types have significant changes between groups. Pearson correlation analysis was used to identify the coexpression pattern between significantly changed immune cells. The results indicated that these immune cells work together and regulate each other (Fig. 7D). Next, we analyzed the relationship between the prognostic characteristics of patients with NSCLC and the immune cell subtypes. As shown in Supplementary Fig. $4 \mathrm{~A}-\mathrm{H}$, the correlation between B cells, $\mathrm{T}$ cells, regulatory $\mathrm{T}$ 
cells (Treg), M2 macrophages, mast cells, dendritic cells (DCs), monocytes, and risk signals was determined.

\subsection{Research on the correlation between immune checkpoint genes and risk score}

The correlation between risk characteristics and immune checkpoint genes was studied in the high-risk and low-risk groups, and the median risk in NSCLC samples was used as the stratification standard. As a result, the expression of PD-1 (Supplementary Fig. 4J), PD-L1 (Supplementary Fig. 4K), CTLA4 (Supplementary Fig. 4L), and PD-L2 (Supplementary Fig. 4I) was relatively higher in the high-risk group.

\section{Discussion}

In our study, we identified and independently verified prognostic signals based on seven ATG IncRNAs (AL691432.2, MMP2-AS1, AC124067.2, CRNDE, ABALON, AL161431.1, and NKILA). GSEA results showed that risk signals were significantly correlated with immune cell function and immune response. This finding led us to further study the relationship between autophagy and immune infiltration.

IncRNAs are a type of noncoding RNA with a length of more than 200 nucleotides. They interact with proteins, RNA, and DNA to regulate gene expression before and after transcription. Studies have found that IncRNAs are widely involved in human life activities such as cell development, differentiation, proliferation, apoptosis, migration, invasion, and metastasis, which are closely related to the formation, progression, and metastasis of malignant tumors. In addition, IncRNA is also involved in chemotherapy resistance and targeted therapy.

A study of endometrial cancer found that IncRNA AL161431.1 targets miR-1252-5p, which leads to the upregulation of MAPK signals and regulates cancer cell apoptosis and autophagy cell death [13]. NKILA is a IncRNA that interacts with NF-KB; it regulates the sensitivity of T cells to T lymphocyte death (AICD) by inhibiting NF-KB activity. NKILA can directly or indirectly inhibit IKBa phosphorylation and NF-KB activation in breast and liver cancers $[14,15]$. In breast cancer studies, inflammatory cytokines were found to upregulate NKILA expression through the NF-KB pathway, which regulates breast cancer metastasis and inhibits angiogenesis in HUVECs $[14,16]$. In nasopharyngeal carcinoma (NPC), NKILA was also found to inhibit NPC metastasis through the NF-KB pathway [17]. P65 in laryngeal cancer tissues can also reportedly positively regulate NKILA expression; however, NKILA can inhibit the transport of P65, thus reducing the resistance of cells to laryngeal cancer X-ray radiation [18]. We found that NKILA reduces MMP14 expression by mediating IKBa phosphorylation and NF-KB translocation to the nucleus, thereby impairing the migration and invasion of ESCC cells [19]. Lung cancer studies have found that NKILA is associated with the survival prognosis of LUAD NKILA expression is regulated by the classic TGF- $\beta$ signaling pathway, which subsequently inhibits the migration and invasion of NSCLC cells by interfering with the NF-KB/Snail signaling pathway in NSCLC cells [20]. A similar function was observed in tongue squamous cell carcinoma [21]. 
Colorectal neoplasia differentially expressed (CRNDE) was first observed in colorectal adenomas and colorectal cancers [22]. CRNDE is elevated in a variety of cancers, including colorectal cancer (CRC) [23, 24], glioma [25], hepatocellular carcinoma [26], and lung cancer [27], and there is increasing evidence that it plays a role in regulating cancer cell proliferation, migration, invasion, and apoptosis. Downregulation of CRNDE reportedly results in the inhibition of CRC cell proliferation and induces cell apoptosis [24]. Ellis et al. found that CRNDE is related to the regulation of aerobic glycolysis, or the Warburg effect, in cancer cells [28]. In lung cancer studies [29], LUAD tissue and CRNDE were significantly associated with poor differentiation, TNM stage, lymph node metastasis, radiotherapy response, and shorter OS time. Liu et al. found that CRNDE promotes NSCLC cell proliferation and growth by activating PI3K/AKT signal transduction [30].

As tumor-infiltrating lymphocytes influence the immunotherapy response as well as the clinical consequences of colorectal, gastric, lung, and breast cancers, their role in various cancer-related processes is increasingly recognized. In this study, we also used gene expression profiles to explore the involvement of TILs in NSCLC patients. In our ATG risk model, the high-risk and low-risk groups, showed a different immune landscape. Specifically, we found that the distribution of B cells, $T$ cells, macrophages, DCs, mast cells, and monocytes in our risk model was different and correlated with risk $(P<0.05)$.

Increasing numbers of studies are using DCs to treat cancer. DCs have strong antigen presentation ability, which may overcome tumor tolerance and induce antitumor immunity [31]. In NSCLC patients, DCs upregulate the expression of the coinhibitory molecule B7-H3 and inhibit T cell activity [32]. Lung tumor cells secrete substances that lead to monocyte-induced DC differentiation disorders, maturation abnormalities, and phenotypic defects. Tumor-induced DC deficiency leads to an insufficient ability to recognize and present tumor antigens and tumor immune escape [33].

Previous studies have shown that T cells are the main immune-infiltrating cells in NSCLC [34]; among these cells, $\mathrm{CD}^{+} \mathrm{T}$ cells are the most abundant $\mathrm{T}$ cell population $(26 \%)$, followed by $\mathrm{CD} 8^{+} \mathrm{T}$ cells $(22 \%)$ [35]. Many studies have shown that low levels of $C D 8^{+} T$ cell infiltration in tumor lesions are associated with poor prognosis [36]. As a key factor in the tumor microenvironment, B cells and neutrophils play an important regulatory role in tumor progression. However, these cells have both antitumor and pro-tumor activities [37-39].

The same macrophages play different roles in the tumor microenvironment. In LUAD, there were higher immune scores, more memory B cells, and more M0 macrophages in the early stage than in the late stage. The abundance of M0 macrophage infiltration was significantly correlated with TNM stage and survival rate [40].

In LUAD, mast cells are associated with tumor angiogenesis and poor prognosis [41, 42]. In this study, a predictive model of autophagy-related IncRNAs was established and validated by univariate and multivariate Cox regression analyses. The value of our $\mathrm{K}-\mathrm{M}$ survival curve was $\mathrm{P}=3.325 \mathrm{e}-05$, indicating that our prediction model was closely related to the survival outcomes of patients with lung cancer. In 
addition, our prognostic model was superior to the other prognostic markers. Finally, we used GSEA to detect the biological functions of the prediction model. These results strongly demonstrate that these IncRNAs are involved in tumor progression and are associated with immune infiltration.

Our study has several limitations. First, our findings need to be further validated in other independent cohorts to determine the stability of autophagy-related IncRNA prognostic markers. Second, our study was based on 712 patients from the publicly available TCGA database. Among them, the samples of NSCLC patients with early cancer $(n=557)$ were significantly larger than those of patients with advanced cancer $(n=155)$, which may have skewed our results; therefore, further analysis with a larger sample size is needed. Finally, further studies on biochemical experiments, such as immunohistochemistry, quantitative real-time polymerase chain reaction, flow cytometry, and clinical data analysis, are needed to confirm our findings.

\section{Conclusions}

In conclusion, we herein constructed an autophagy-related IncRNA model of NSCLC. These findings suggest that seven prognostic models of autophagy-related IncRNAs can effectively predict clinical prognosis. In addition, based on the effect of the immune-infused microenvironment on tumor proliferation and metastasis, our findings provide a new direction for immunotherapy.

\section{Declarations}

\section{Data availability statements}

The RNA-Seq FPKM data were downloaded from the TCGA GDC data portal (https://portal.gdc.cancer.gov/), and its code was TCGA-LUAD and TCGA-LUSC. ATGs were downloaded from the Human Autophagy Database (http://www.autophagy.lu/).

\section{Acknowledgements}

We acknowledge TCGA database for providing their platforms and contributors for uploading their meaningful datasets. We thank Professor Wenning Wu for providing guidance.

\section{Funding}

The fund for the Natural Science Foundation of China (No. 81970051), Excellent Top Talent Cultivation Project of Anhui Higher Education Institutions (gxyqZD2017030), the fund from Reserve candidate for

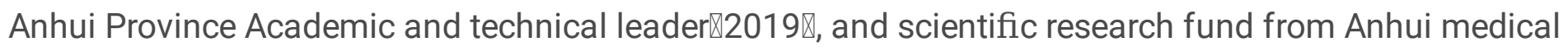
university (2020xkj257) supported this research.

\section{Author contributions}


S.Z. and R.W. conceived and designed the experiments. H.J., A.X., M.L., R.H., E.W. and K.Z. analyzed data. H.J. and R.W. wrote this manuscript. All authors read and approved the final manuscript.

\section{Ethics approval and consent to participate}

Not applicable

\section{Patient consent for publication}

Not apolicable.

\section{Declaration of Competing Interest}

The authors declare that they have no competing interests.

\section{References}

1. Siegel RL, Miller KD, Jemal A. Cancer statistics, 2016[J]. CA Cancer J Clin. 2016;66(1):7-30.

2. Global Burden of Disease. Cancer C, Fitzmaurice C, Abate D, et al. Global, Regional, and National Cancer Incidence, Mortality, Years of Life Lost, Years Lived With Disability, and Disability-Adjusted Life-Years for 29 Cancer Groups, 1990 to 2017: A Systematic Analysis for the Global Burden of Disease Study[J]. JAMA Oncol, 2019, 5(12): 1749-68.

3. Petroziello J, Yamane A, Westendorf $L$, et al. Suppression subtractive hybridization and expression profiling identifies a unique set of genes overexpressed in non-small-cell lung cancer[J]. Oncogene. 2004;23(46):7734-45.

4. Gutschner $T$, Hammerle M, Eissmann $M$, et al. The noncoding RNA MALAT1 is a critical regulator of the metastasis phenotype of lung cancer cells[J]. Cancer Res. 2013;73(3):1180-9.

5. Mizushima N, Komatsu M. Autophagy: renovation of cells and tissues[J]. Cell. 2011;147(4):728-41.

6. Levine B, Kroemer G. Autophagy in the pathogenesis of disease[J]. Cell. 2008;132(1):27-42.

7. Maiuri MC, Zalckvar E, Kimchi A, et al. Self-eating and self-killing: crosstalk between autophagy and apoptosis[J]. Nat Rev Mol Cell Biol. 2007;8(9):741-52.

8. Galluzzi L, Pietrocola F, Bravo-San Pedro JM, et al. Autophagy in malignant transformation and cancer progression[J]. EMBO J. 2015;34(7):856-80.

9. Guo JY, White E. Autophagy, Metabolism, and Cancer[J]. Cold Spring Harb Symp Quant Biol. 2016;81:73-8.

10. White E, Dipaola RS. The double-edged sword of autophagy modulation in cancer[J]. Clin Cancer Res. 2009;15(17):5308-16.

11. Degenhardt $K$, Chen $G$, Lindsten $T$, et al. BAX and BAK mediate $p 53$-independent suppression of tumorigenesis [J]. Cancer Cell. 2002;2(3):193-203. 
12. Xia Z, Ou-Yang W, Hu T. Prognostic significance of CDC25C in lung adenocarcinoma: An analysis of TCGA data[J]. Cancer Genet, 2019, 233-234: 67-74.

13. Gu ZR, Liu W. The LncRNA AL161431.1 targets miR-1252-5p and facilitates cellular proliferation and migration via MAPK signaling in endometrial carcinoma. Eur Rev Med Pharmacol Sci. 2020;24(5):2294-302.

14. Liu B, Sun L, Liu Q, et al. A cytoplasmic NF-kappaB interacting long noncoding RNA blocks IkappaB phosphorylation and suppresses breast cancer metastasis[J]. Cancer Cell. 2015;27(3):370-81.

15. Yu X, Tang W, Yang Y, et al. Long noncoding RNA NKILA enhances the anti-cancer effects of baicalein in hepatocellular carcinoma via the regulation of NF-kappaB signaling[J]. Chem Biol Interact. 2018;285:48-58.

16. Luo LH, Rao L, Luo LF, et al. Long non-coding RNA NKILA inhibited angiogenesis of breast cancer through NF-kappaB/IL-6 signaling pathway[J]. Microvasc Res. 2020;129:103968.

17. Zhang W, Guo Q, Liu G, et al. NKILA represses nasopharyngeal carcinoma carcinogenesis and metastasis by NF-kappaB pathway inhibition[J]. PLoS Genet. 2019;15(8):e1008325.

18. Yang T, Li S, Liu J, et al. IncRNA-NKILA/NF-kappaB feedback loop modulates laryngeal cancer cell proliferation, invasion, and radioresistance[J]. Cancer Med. 2018;7(5):2048-63.

19. Lu Z, Chen Z, Li Y, et al. TGF-beta-induced NKILA inhibits ESCC cell migration and invasion through NF-kappaB/MMP14 signaling[J]. J Mol Med (Berl). 2018;96(3-4):301-13.

20. Liu D, Shi X. Long non-coding RNA NKILA inhibits proliferation and migration of lung cancer via IL11/STAT3 signaling [J]. Int J Clin Exp Pathol. 2019;12(7):2595-603.

21. Huang W, Cui $X$, Chen J, et al. Long non-coding RNA NKILA inhibits migration and invasion of tongue squamous cell carcinoma cells via suppressing epithelial-mesenchymal transition[J]. Oncotarget. 2016;7(38):62520-32.

22. Graham LD, Pedersen SK, Brown GS, et al. Colorectal Neoplasia Differentially Expressed (CRNDE), a Novel Gene with Elevated Expression in Colorectal Adenomas and Adenocarcinomas[J]. Genes Cancer. 2011;2(8):829-40.

23. Liu T, Zhang X, Yang YM, et al. Increased expression of the long noncoding RNA CRNDE-h indicates a poor prognosis in colorectal cancer, and is positively correlated with IRX5 mRNA expression[J]. Onco Targets Ther. 2016;9:1437-48.

24. Ding J, Li J, Wang $\mathrm{H}$, et al. Long noncoding RNA CRNDE promotes colorectal cancer cell proliferation via epigenetically silencing DUSP5/CDKN1A expression[J]. Cell Death Dis. 2017;8(8):e2997.

25. Zhang X, Sun S, Pu JK, et al. Long non-coding RNA expression profiles predict clinical phenotypes in glioma[J]. Neurobiol Dis. 2012;48(1):1-8.

26. Chen Z, Yu C, Zhan L, Pan Y, Chen L, Sun C. LncRNA CRNDE promotes hepatic carcinoma cell proliferation, migration and invasion by suppressing miR-384[J]. Am J Cancer Res. 2016;6(10):2299-309. 
27. Jin $B$, Wang W, Du G, et al. Identifying hub genes and dysregulated pathways in hepatocellular carcinoma [J]. Eur Rev Med Pharmacol Sci. 2015;19(4):592-601.

28. Ellis BC, Graham LD, Molloy PL. CRNDE, a long non-coding RNA responsive to insulin/IGF signaling, regulates genes involved in central metabolism[J]. Biochimica et Biophysica Acta (BBA). - Molecular Cell Research. 2014;1843(2):372-86.

29. Zhang M, Gao C, Yang Y, et al. Long Noncoding RNA CRNDE/PRC2 Participated in the Radiotherapy Resistance of Human Lung Adenocarcinoma Through Targeting p21 Expression[J]. Oncol Res. 2018;26(8):1245-55.

30. Liu XX, Xiong HP, Huang JS, et al. Highly expressed long non-coding RNA CRNDE promotes cell proliferation through PI3K/AKT signalling in non-small cell lung carcinoma[J]. Clin Exp Pharmacol Physiol. 2017;44(8):895-902.

31. Sabado RL, Bhardwaj N. Dendritic-cell vaccines on the move[J]. Nature. 2015;519(7543):300-1.

32. Schneider T, Hoffmann $\mathrm{H}$, Dienemann $\mathrm{H}$, et al. Non-small cell lung cancer induces an immunosuppressive phenotype of dendritic cells in tumor microenvironment by upregulating B7-H3 [J]. J Thorac Oncol. 2011;6(7):1162-8.

33. Youlin K, Weiyang H, Simin L, et al. Prostaglandin E2 Inhibits Prostate Cancer Progression by Countervailing Tumor Microenvironment-Induced Impairment of Dendritic Cell Migration through LXRalpha/CCR7 Pathway[J]. J Immunol Res, 2018, 2018: 5808962.

34. Winter $H$, Van Den Engel NK, Ruttinger $D$, et al. Therapeutic $T$ cells induce tumor-directed chemotaxis of innate immune cells through tumor-specific secretion of chemokines and stimulation of B16BL6 melanoma to secrete chemokines[J]. J Transl Med. 2007;5:56.

35. Stankovic B, Bjorhovde H, Skarshaug K. R, et al. Immune Cell Composition in Human Non-small Cell Lung Cancer[J]. Front Immunol. 2018;9:3101.

36. Ma J, Zheng B, Goswami S, et al. PD1(Hi) CD8(+) T cells correlate with exhausted signature and poor clinical outcome in hepatocellular carcinoma[J]. J Immunother Cancer. 2019;7(1):331.

37. Andreu P, Johansson $\mathrm{M}$, Affara NI, et al. FcRgamma activation regulates inflammation-associated squamous carcinogenesis[J]. Cancer Cell. 2010;17(2):121-34.

38. Fujimoto M, Yoshizawa A, Sumiyoshi S, et al. Stromal plasma cells expressing immunoglobulin G4 subclass in non-small cell lung cancer[J]. Hum Pathol. 2013;44(8):1569-76.

39. Fridlender ZG, Sun J, Kim S, et al. Polarization of tumor-associated neutrophil phenotype by TGFbeta: "N1" versus "N2" TAN[J]. Cancer Cell. 2009;16(3):183-94.

40. Tamminga M, Hiltermann TJN, Schuuring $E$, et al. Immune microenvironment composition in nonsmall cell lung cancer and its association with survival[J]. Clin Transl Immunology. 2020;9(6):e1142.

41. Theoharides TC, Conti P. Mast cells: the Jekyll and Hyde of tumor growth[J]. Trends Immunol. 2004;25(5):235-41.

42. Ch'ng S, Wallis RA, Yuan L, et al. Mast cells and cutaneous malignancies[J]. Mod Pathol. 2006;19(1):149-59. 

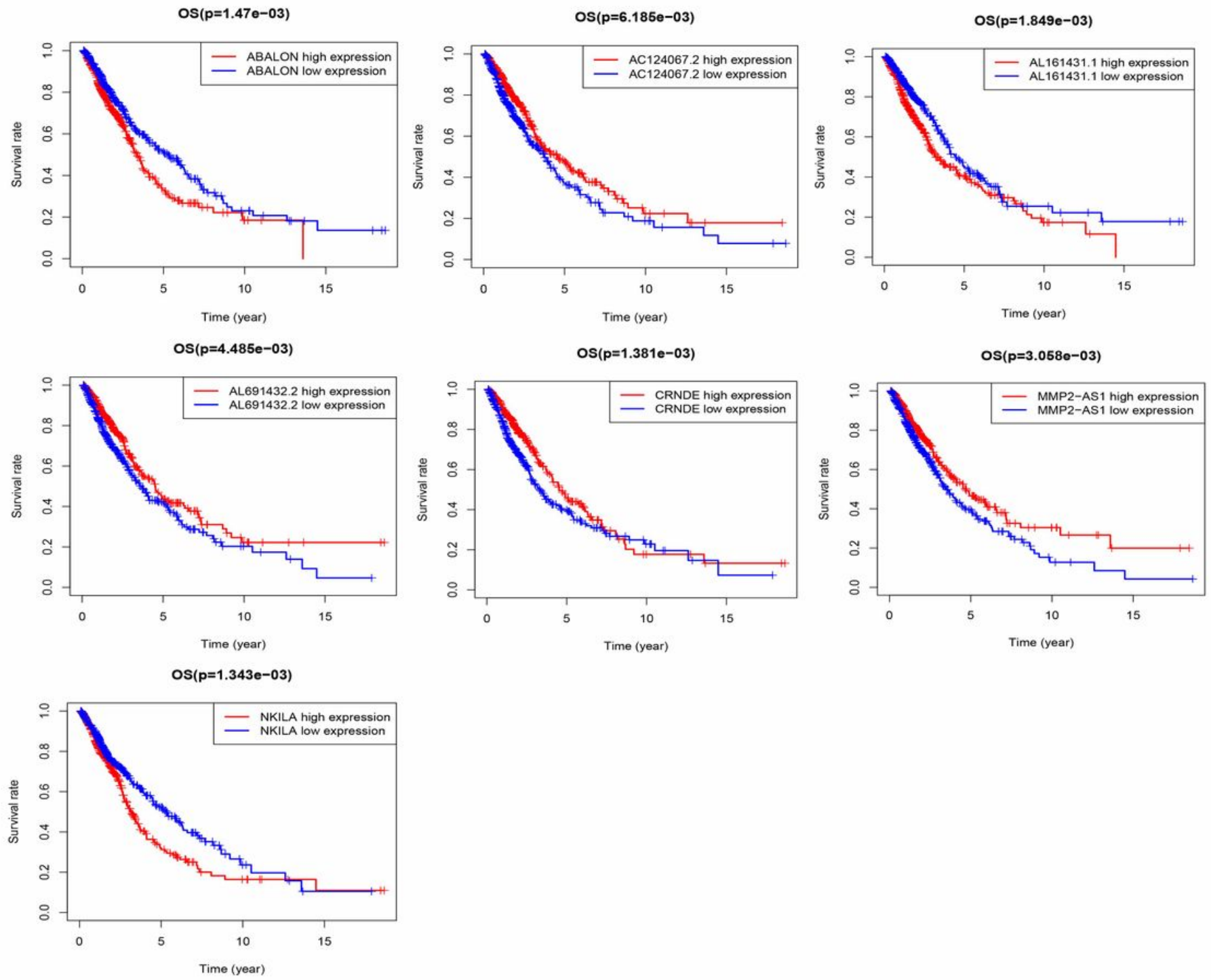

\section{Figure 1}

Construction of prognostic markers related to autophagy of IncRNA in non-small cell lung cancer (NSCLC) patients. Overall survival curves of seven autophagy-related IncRNAs in The Cancer Genoma Atlas (TCGA) database (A-G). 
A
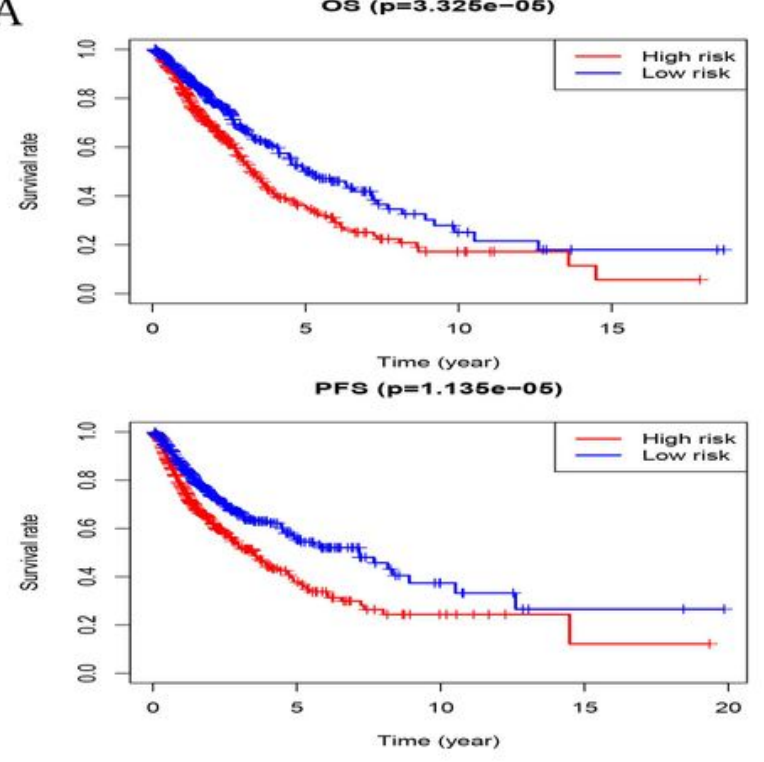

C

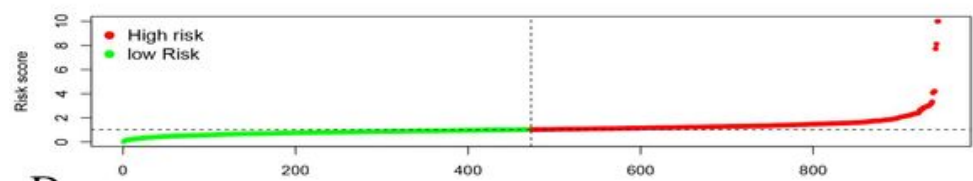

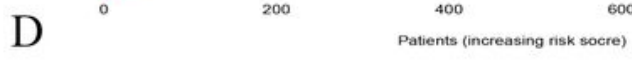

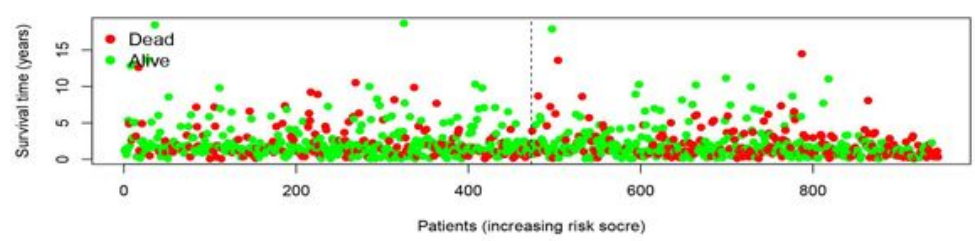

E

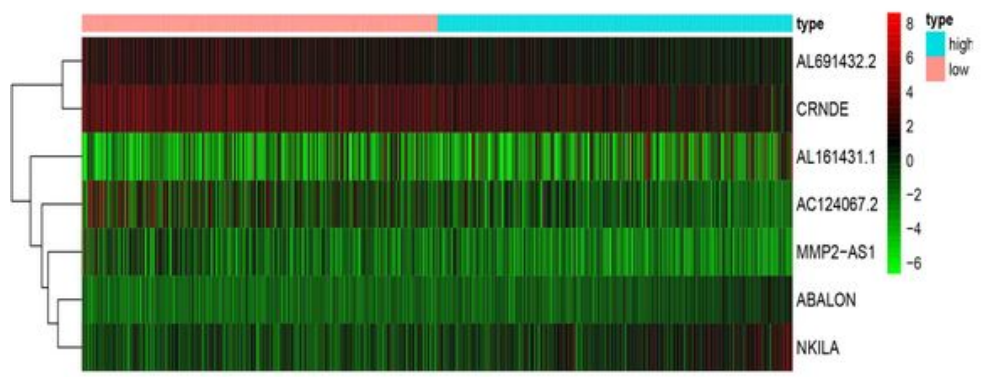

B

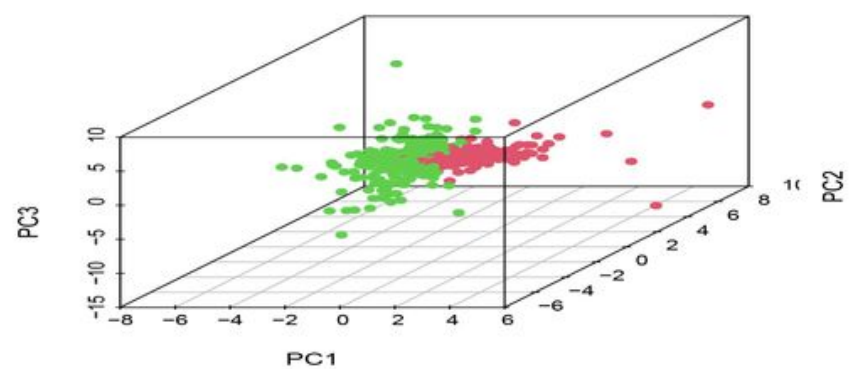

$\mathrm{F}$

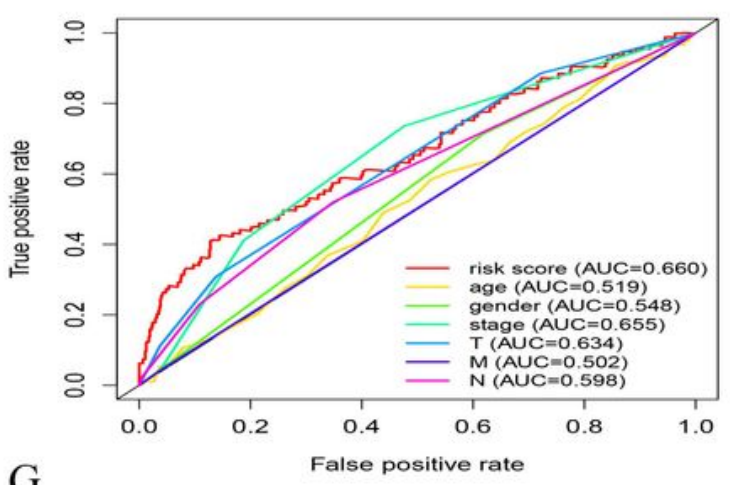

G
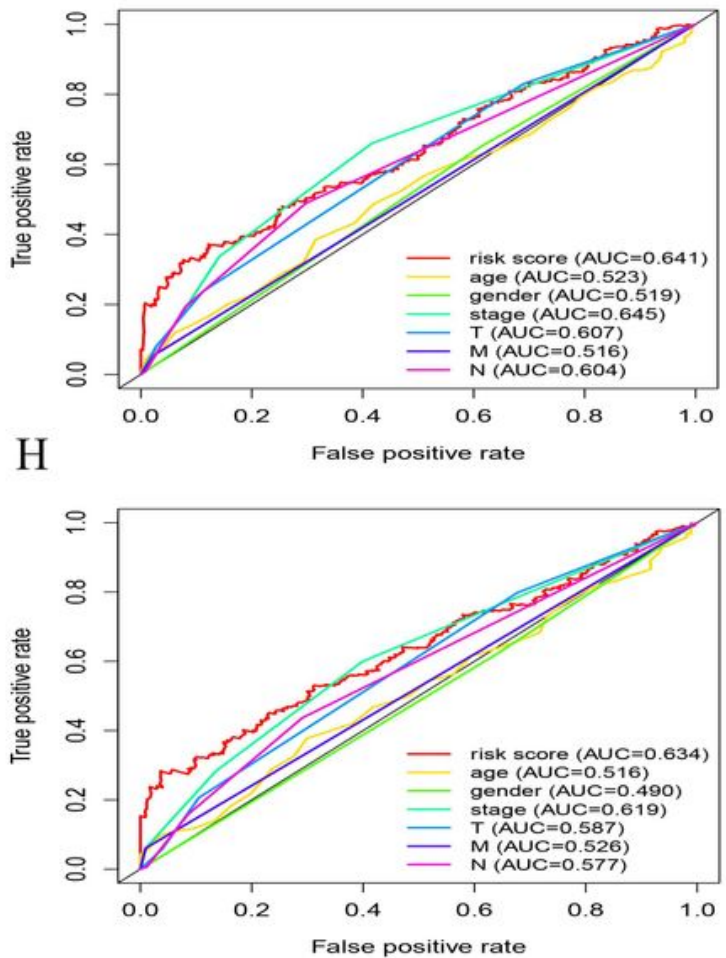

\section{Figure 2}

Validation of prognostic markers related to autophagy of IncRNA in non-small cell lung cancer (NSCLC) patients. (A) The Kaplan-Meier survival curve for patients with high/low risk scores for autophagy-related IncRNA prognostic characteristics. (B) Principal component analysis (PCA) was performed according to seven autophagy-related IncRNAs, showing two significantly different distribution patterns in the high-risk and low-risk groups. (C) Distribution of risk scores in high-risk and low-risk NSCLC patients based on 
prognostic features of autophagy-related IncRNA. (D) Autophagy-related IncRNA prognostic characteristics of NSCLC patients with survival time and risk score. (E) Expression of autophagy-related IncRNA in high-risk and low-risk patients. $(\mathrm{F}-\mathrm{H})$ Receiver operating characteristic (ROC) curves evaluating the accuracy of each factor for predicting the (F) 1-year, (G) 3-year, and $(\mathrm{H})$ 5-year survival.
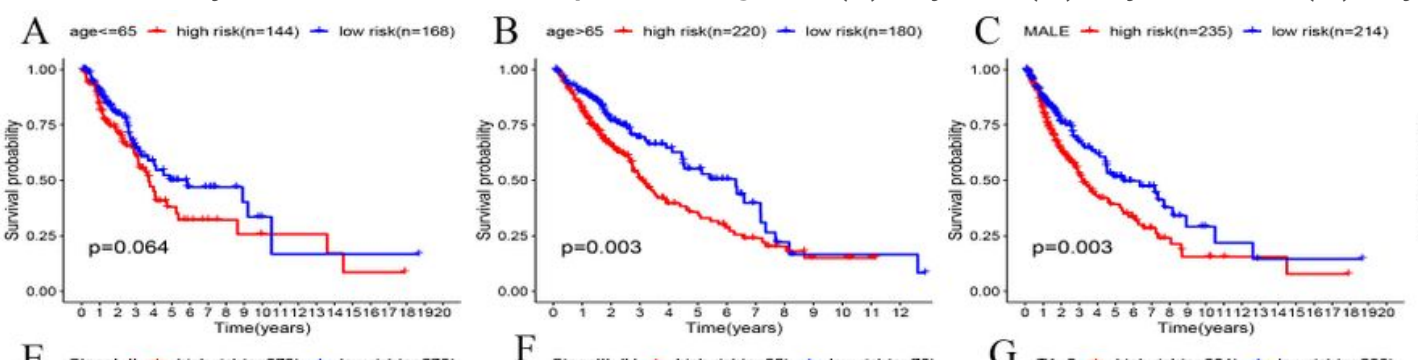

D FEMALE - high risk(n=129) - low risk(n=134)
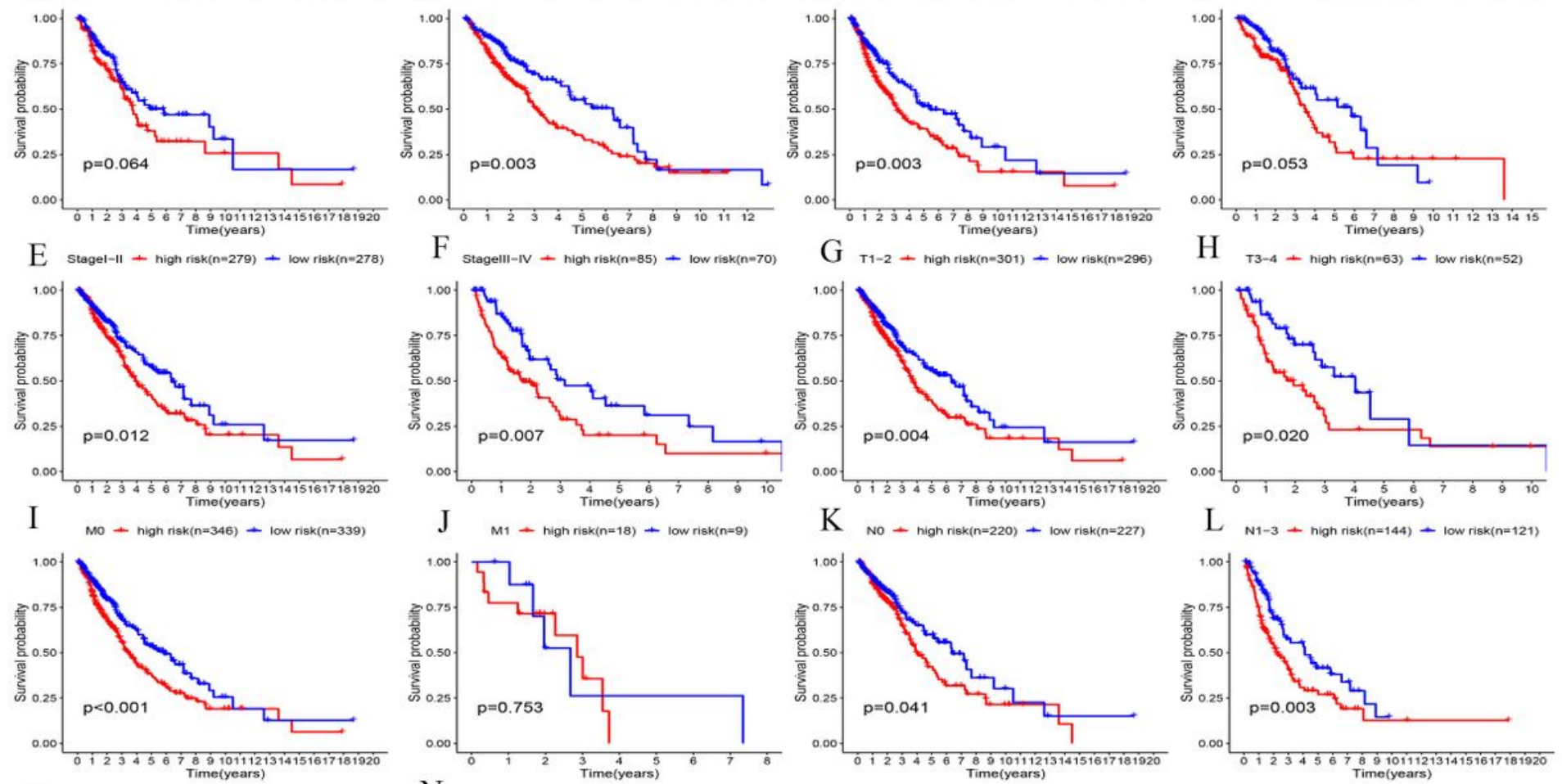

M LUAD - high risk(n=176) - low risk(n=148)
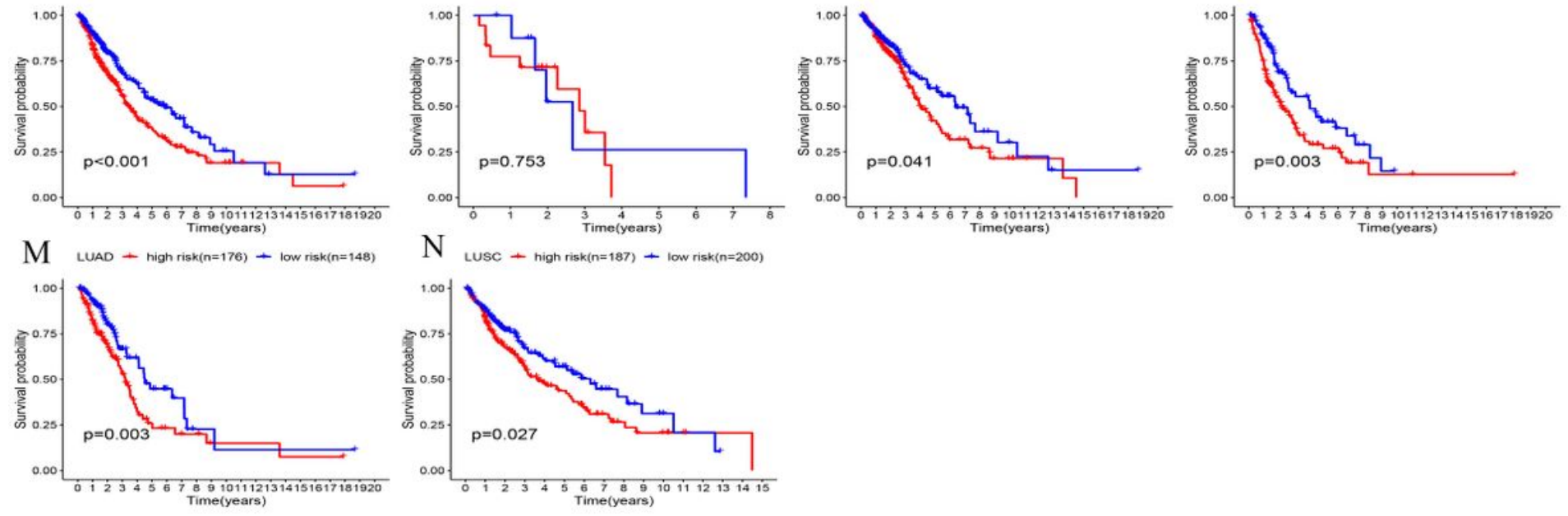

\section{Figure 3}

Overall survival (OS) rates of high-risk and low-risk non-small cell lung cancer (NSCLC) patients stratified by clinicopathological features. Kaplan-Meier survival curve analysis showed that the OS of patients with high-risk and low-risk NSCLC in The Cancer Genome Atlas (TCGA) database was classified as (A, B) age ( $\leq 65$ years vs. $>65$ years), (C, D) sex (male vs. female), (E, F) AJCC stages (stages I and II vs. stages III and IV), (G, H) T stages (T1/T2 vs. T3/T4), (I, J) M stage (M0 vs. M1), (K, L) N stages (N0 vs. N1/N2/N3) stages, and $(\mathrm{M}, \mathrm{N})$ pathological type (lung adenocarcinoma [LUAD] vs. lung squamous cell carcinoma [LUSC]). 


\begin{tabular}{|c|c|c|}
\hline & pvalue & Hazard ratio \\
\hline age & 0.286 & $1.007(0.994-1.021)$ \\
\hline gender & 0.480 & $1.097(0.849-1.416)$ \\
\hline stage & $<0.001$ & $1.485(1.310-1.683)$ \\
\hline $\mathrm{T}$ & $<0.001$ & $1.424(1.225-1.656)$ \\
\hline M & 0.020 & $1.859(1.102-3.137)$ \\
\hline $\mathrm{N}$ & $<0.001$ & $1.478(1.273-1.717)$ \\
\hline riskScore & $<0.001$ & $1.193(1.140-1.248)$ \\
\hline
\end{tabular}

$\mathrm{B}$

$\begin{array}{lll}\text { stage } & 0.100 & 1.288(0.953-1.741) \\ \mathrm{T} & 0.088 & 1.185(0.975-1.439) \\ \mathrm{M} & 0.943 & 1.029(0.467-2.265) \\ \mathrm{N} & 0.247 & 1.167(0.898-1.517)\end{array}$

riskScore $<0.0011 .191(1.137-1.247)$

\section{pvalue Hazard ratio}
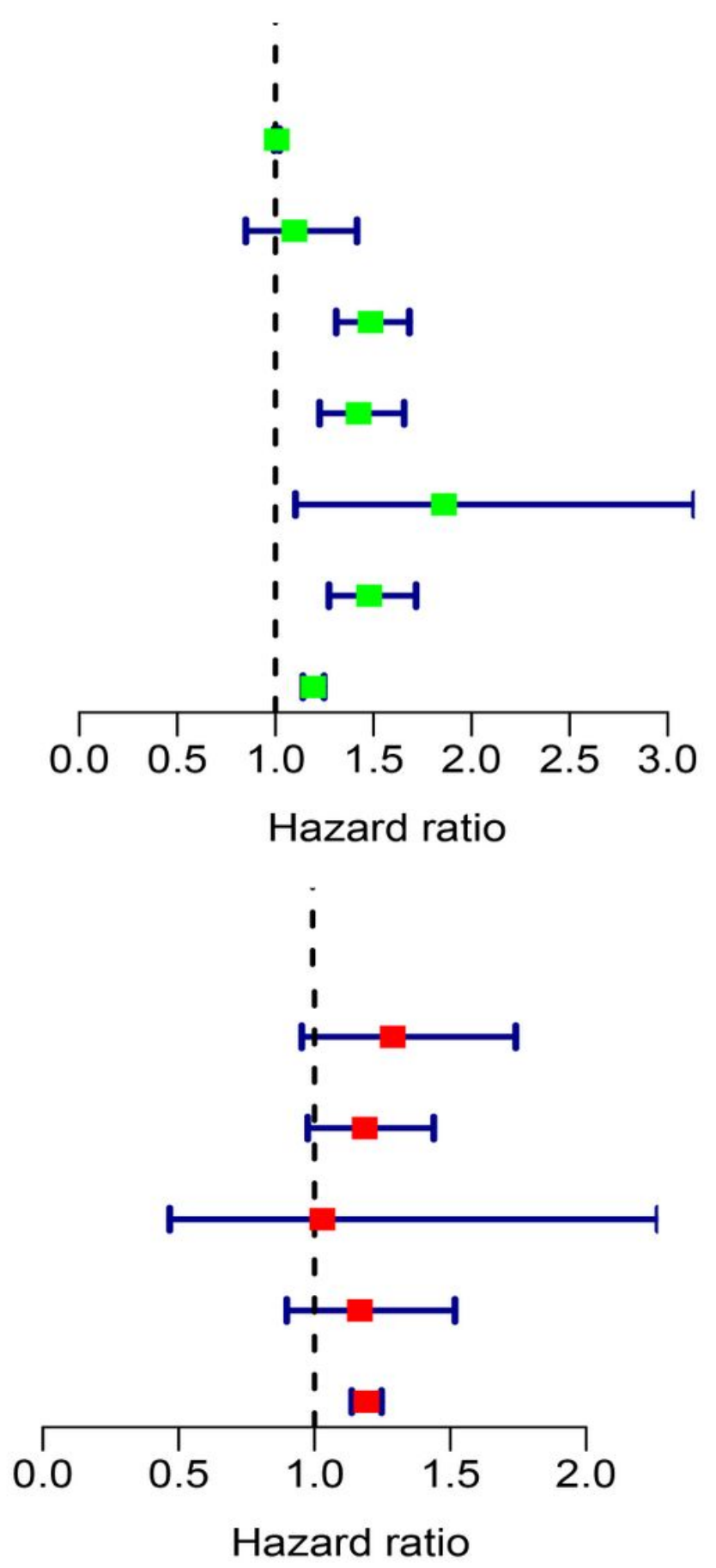

Figure 4

To evaluate prognostic characteristics of autophagy-related IncRNA and prognostic accuracy of other clinicopathological variables in non-small cell lung cancer (NSCLC) patients. (A) Univariate Cox regression analysis; (B) Multivariate independent prognostic analysis. 
A

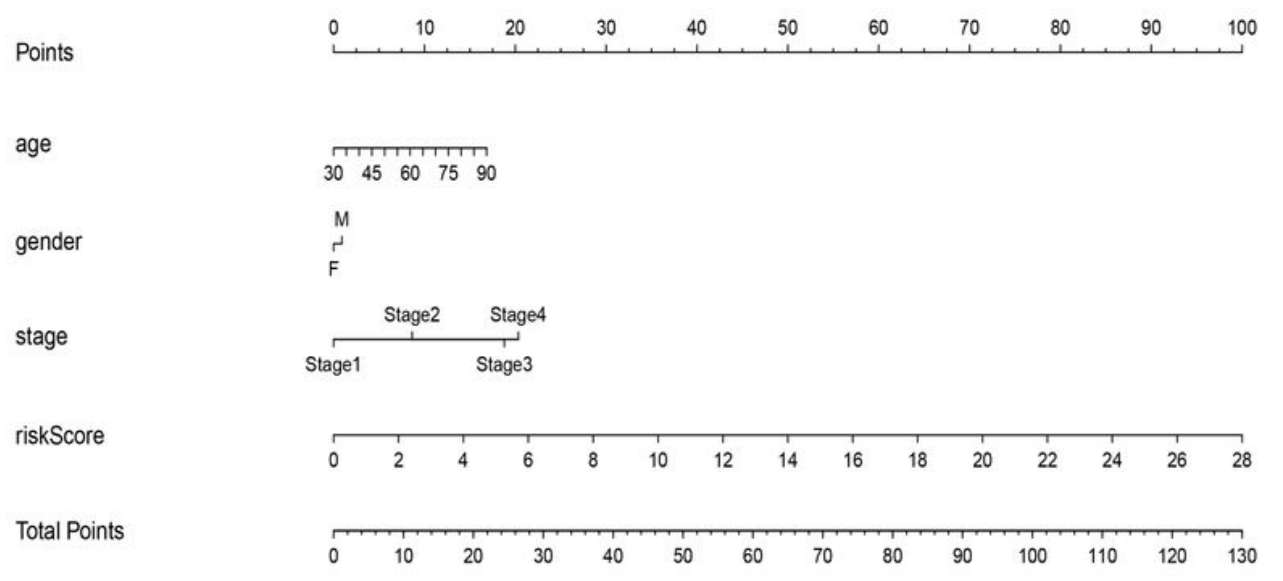

1-year survival

3-year survival

5-year survival

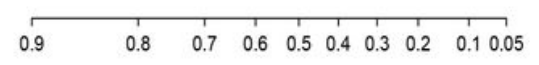

\begin{tabular}{lllllllll}
\hline 0.8 & 0.7 & 0.6 & 0.5 & 0.4 & 0.3 & 0.2 & 0.1 & 0.05
\end{tabular}

\begin{tabular}{lllllllll}
\hline 0.8 & 0.7 & 0.6 & 0.5 & 0.4 & 0.3 & 0.2 & 0.1 & 1 \\
\hline
\end{tabular}

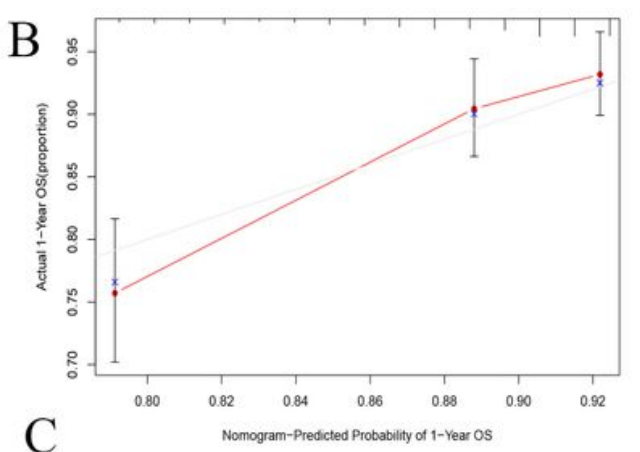

$\mathrm{C}$

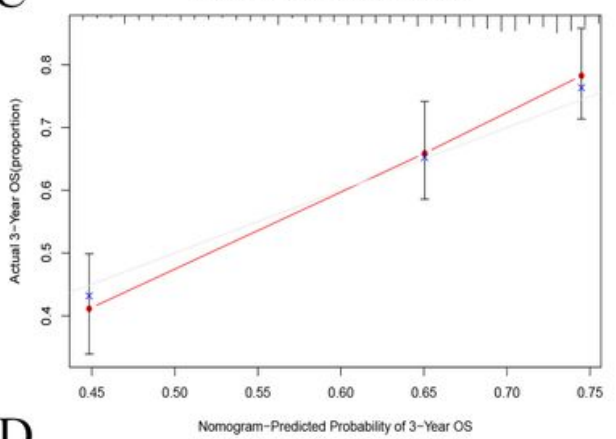

$\mathrm{D}$

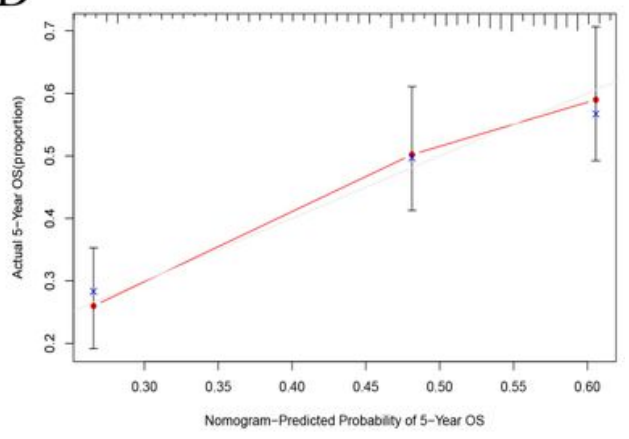

\section{Figure 5}

Construction and validation of a prognostic nomogram using autophagy-associated IncRNA prognostic characteristic risk score as a parameter. (A) A prognostic nomogram was constructed based on the prognostic characteristics of autophagy-related IncRNA and risk scores of clinicopathological parameters such as age, sex, AJCC stage, T stage, and N stage. The nomogram shows the predicted 1-, 3-, and 5-year survival rates of NSCLC patients. The correction curve showed consistency between predicted and the (B) 1-year, (C) 3-year, and (D) 5-year survival rates for high-risk and low-risk BCLA patients. 
A

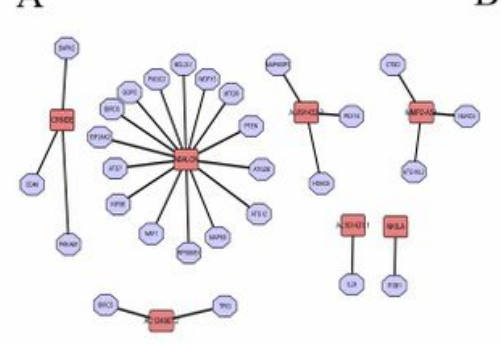

C
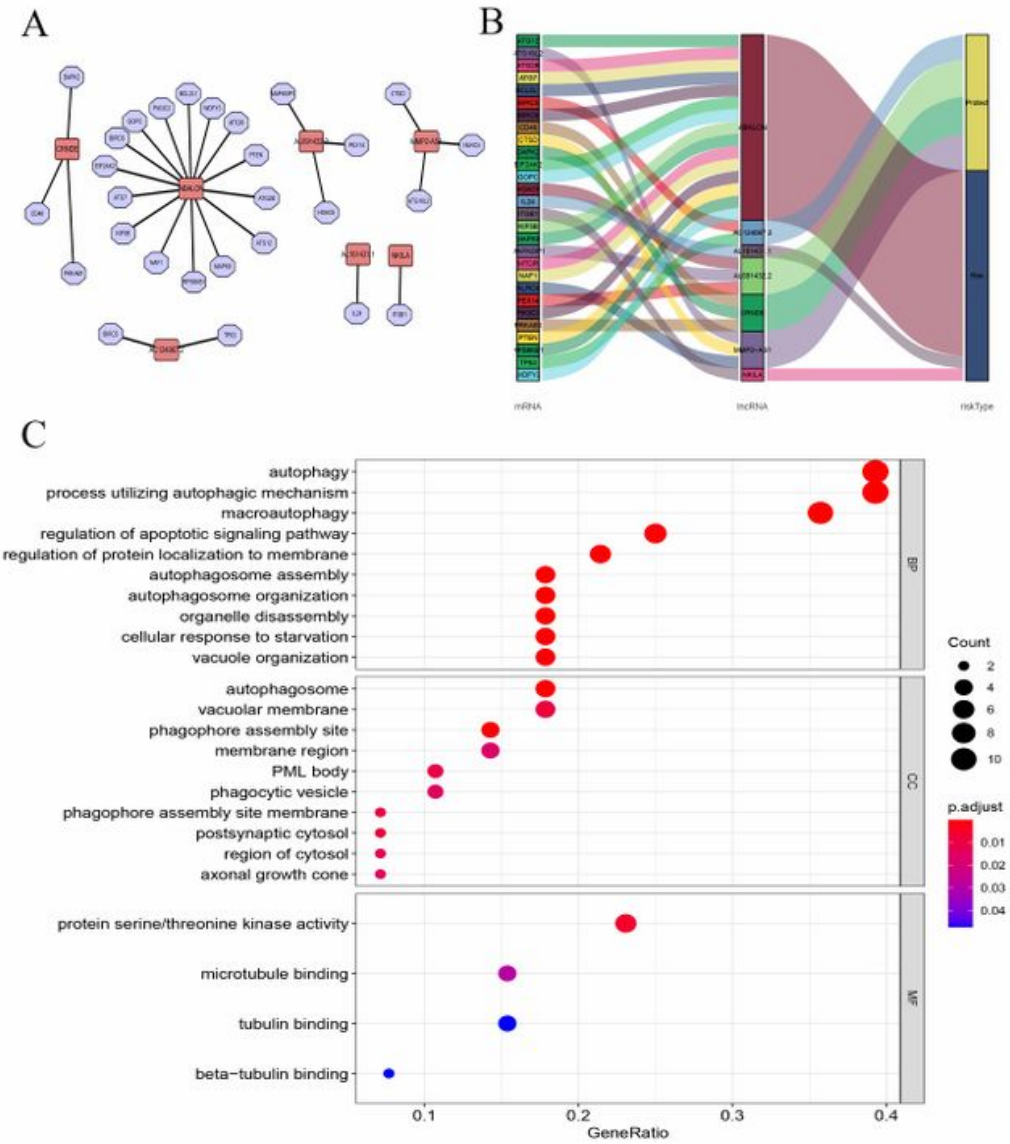

D

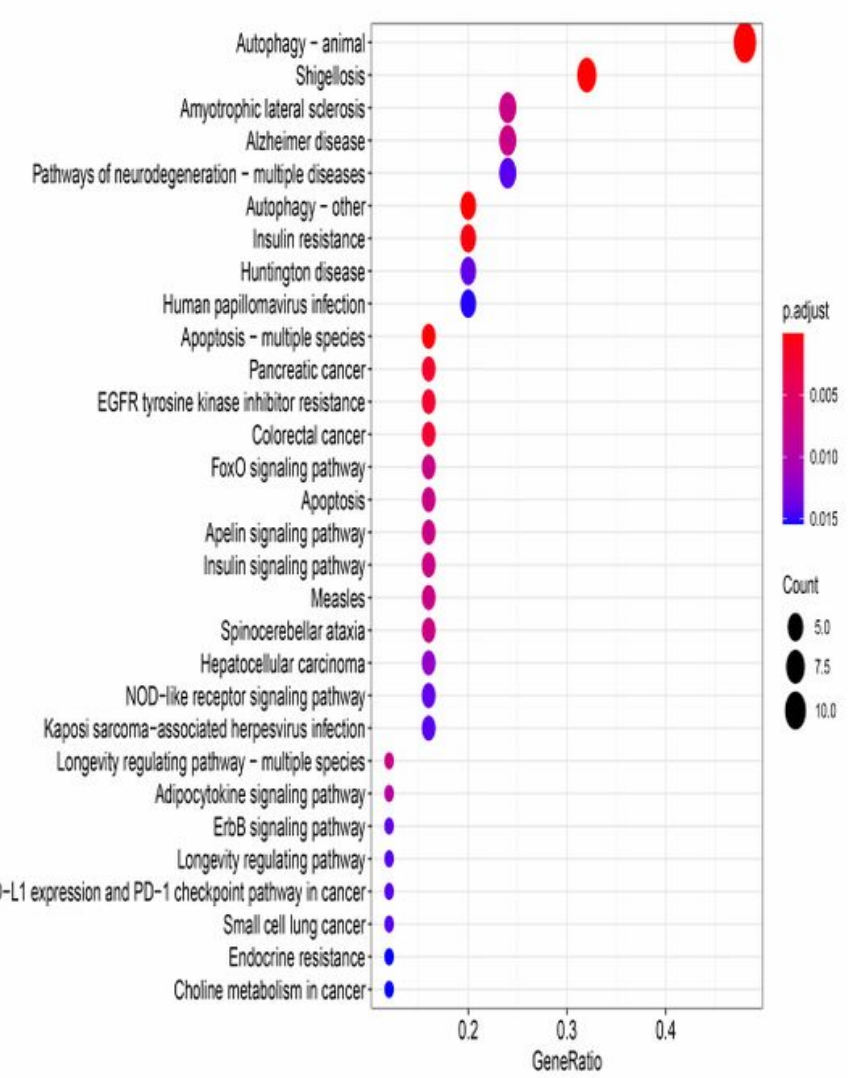

\section{Figure 6}

Construction and functional enrichment analysis of the autophagy-related IncRNA-mRNA coexpression network. (A) Network diagram of macrophage-related IncRNA-mRNA. The red circle corresponds to autophagy-related IncRNA, and the purple circle corresponds to mRNA. (B) The Sankey diagram showed the degree of connection between 28 mRNAs and seven autophagy-related IncRNAs (risk/protective). (C) The results of Gene Ontology (GO) analysis showed the biological process, cellular components, and molecular functions of mRNA enrichment coexpressed with five autophagy-related IncRNAs. (D) Results of the Kyoto Encyclopedia of Genes and Genomes (KEGG) pathway analysis show that the mRNA genomes coexpressed with seven autophagy-related IncRNAs are in the enriched signaling pathways. 

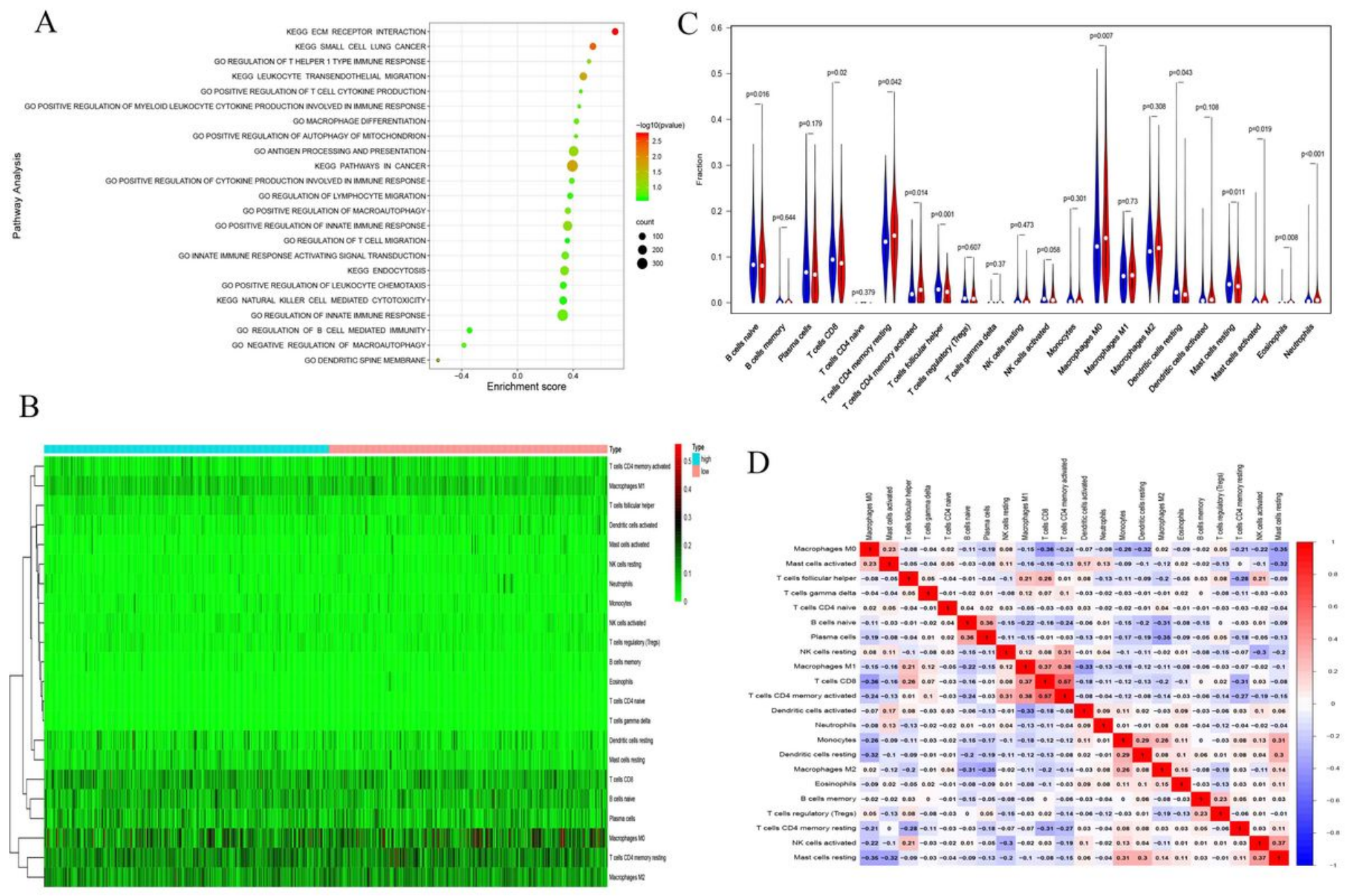

Figure 7

Relationship between autophagy-related gene (ATG) risk characteristics and tumor immune microenvironment. (A) Based on the prognostic characteristics of autophagy-related IncRNA, gene set enrichment analysis (GSEA) was performed for high-risk and low-risk non-small cell lung cancer (NSCLC) patients. A bubble diagram showed that risk signals were closely related to the functions and pathways associated with immune cell responses $(P<0.25)$. (B) The heat map shows the normalized absolute abundance of each immune cell type in an individual sample. (C) The violin plot shows the differential expression of immune cells in the high-risk and low-risk groups stratified by risk characteristics. (D) The correlational heat map shows significantly altered gene coexpression patterns between immune cells.

\section{Supplementary Files}

This is a list of supplementary files associated with this preprint. Click to download.

- supplementaryfigure1.jpg

- supplementaryfigure2.jpg

- supplementaryfigure3.jpg 
- supplementaryfigure4.jpg

- Supplementarytable.docx 